\title{
EDITORIAL
}

\section{Peripartum anxiety: parsing heterogeneity in clinical settings}

\author{
Verinder Sharma ${ }^{1,2}$ iD \\ ${ }^{1}$ Department of Psychiatry and Department of Obstetrics and Gynecology, Western University, London, ON, Canada. ${ }^{2}$ Parkwood Institute \\ Mental Health Care Building, London, ON, Canada.
}

A recent meta-analysis estimated that $20.7 \%$ of women have one or more anxiety disorders during pregnancy or in the postpartum period. ${ }^{1}$ Another meta-analysis reported that an estimated $8.5 \%$ of postpartum women have one or more anxiety disorders. ${ }^{2}$ In spite of its common occurrence, the detection and diagnosis of peripartum anxiety disorders can be challenging for several reasons. Assessing anxiety requires consideration of several core anxiety and related disorders. Anxiety disorders are usually accompanied by other psychiatric disorders, particularly major depression. Consequently, anxiety in clinical settings may be considered a symptom of major depressive disorder rather than a co-occurring disorder. Finally, a lack of comprehensive screening may lead to underdiagnosis and sub-optimal treatment of anxiety disorders. This editorial discusses the heterogeneity of peripartum anxiety followed by suggestions for its screening, diagnosis and treatment.

As shown in Box 1, anxiety can occur in the context of core disorders such as panic disorder, agoraphobia, generalized anxiety disorder, social phobia, or specific phobia. Anxiety can also be a manifestation of related disorders, such as obsessive-compulsive disorder, posttraumatic stress disorder, acute stress disorder, or illness anxiety disorder. Anxiety can accompany major depressive disorder or bipolar disorder in the form of anxious distress. Comorbidity with other anxiety or related disorders is also common. For example, approximately $90 \%$ of women with childbirth-related post-traumatic disorder have postpartum depression, and $31 \%$ of women with postpartum depression have childbirth-related post-traumatic stress disorder. ${ }^{3}$ Another study found that approximately $70 \%$ of women who screened positive for obsessive-compulsive disorder also screened positive for postpartum depression. ${ }^{4}$

Peripartum anxiety generally occurs in the context of a pre-existing psychiatric disorder. However, first onset of obsessive-compulsive disorder is not uncommon. Pregnancy appears to be a specific trigger for the onset or exacerbation of obsessive-compulsive disorder or panic disorder. Despite its common occurrence during or

Correspondence: Verinder Sharma, Parkwood Institute Mental Health Care Building, P.O. Box 5777, Stn B, London, ON N6A 4V2, Canada.

E-mail: vsharma@uwo.ca

Submitted Apr 11 2021, accepted Apr 14 2021, Epub May 212021. after pregnancy, obsessive-compulsive disorder may be frequently underdiagnosed or misdiagnosed. Due to the distressing nature of obsessive thoughts or images, women may feel uncomfortable or ashamed about reporting these symptoms. Illness anxiety disorder, a related somatic symptom disorder, is considered a variant of obsessive-compulsive disorder by some researchers. It manifests in the form of anxiety about acquiring or having a serious physical illness. We have recently reported that various reproductive events, including pregnancy and the postpartum period, are associated with increased risk of onset or exacerbation of illness anxiety disorder. Unless asked specifically about the source of anxiety, women do not generally disclose their fears about serious physical illness. Tokophobia, described as an irrational fear and avoidance of childbirth in spite of desperately wanting a child, is common, particularly in nulliparous women.

A study with a primarily female sample found that $78 \%$ of patients with major depressive disorder met the DSM-5 criteria for anxious distress specifier. Symptoms of anxious distress, such as feeling tense, restlessness, and a feeling that something bad will happen, may be particularly common in the peripartum population. ${ }^{5}$ The clinical significance of the specifier lies in its association with a more severe course of illness, worse outcomes, and an increased risk of self-harm.

Women are generally not screened for anxiety during or after pregnancy. Among the screening instruments, there is considerable variation regarding the assessed constructs. Some studies have focused on identifying general anxiety, others on pregnancy-related anxiety, and yet others on specific anxiety disorders. Only a few studies have validated the performance of scales against a structured diagnostic interview. The National Institute for Health and Care Excellence guidelines on perinatal mental health recommend using the 2-item Generalized Anxiety Disorder scale (GAD-2) for screening in pregnant or postpartum women. The GAD-2, a reasonable option for screening in the general population, has a sensitivity of $86 \%$ and specificity of $83 \%$ using a cut-off score of 3 . However, there are scant data on its use in the perinatal

How to cite this article: Sharma V. Peripartum anxiety: parsing heterogeneity in clinical settings. Braz J Psychiatry. 2022;44:4-5. http://dx.doi.org/10.1590/1516-4446-2021-1952 
Box 1 DSM-5 core anxiety and related disorders during pregnancy and postpartum

Core anxiety disorders ${ }^{\dagger} \quad$ Panic disorder, agoraphobia, generalized anxiety disorder, social phobia and specific phobia

Related disorders Bipolar I or II disorders with anxious distress, major depressive disorder with anxious distress, obsessive-compulsive disorder, posttraumatic stress disorder, acute stress disorder and illness anxiety disorder

${ }^{\dagger}$ Excluding substance/medication-induced anxiety disorder and anxiety disorder due to another medical condition.

population. Moreover, a recent study concluded that neither the GAD-2 nor the Edinburgh Postnatal Depression Scale can be recommended for widespread screening of peripartum anxiety. In general, screening tools that assess core anxiety and related disorders should be preferred over measures of general anxiety.

Women with a severe anxiety disorder, comorbid anxiety and depression, or those at risk of harming themselves or the newborn should be referred for psychiatric evaluation and follow-up. Assessment should include exploration of anxiety symptoms (anxious distress), anxiety disorders, and common comorbidities, such as mood disorders or substance use disorder. Women should be specifically asked about the symptoms of obsessivecompulsive disorder or illness anxiety disorder. An organic cause of anxiety should be suspected when the patient has signs or symptoms suggestive of preeclampsia, hyperthyroidism, or substance use/abuse or withdrawal. Suicide risk assessment should be an integral part of the evaluation of women with anxiety, particularly those with comorbid mood disorders.

Correct diagnosis of constituent disorders is a prerequisite to proper management of peripartum anxiety. Treatment strategies should target the underlying psychiatric disorder rather than manage anxiety symptoms. Drug treatment is indicated for cases of severe anxiety or for those with anxiety and comorbid depression. Antidepressants should be avoided in women with anxiety and comorbid bipolar depression, and preference should be given to mood stabilizers such as lamotrigine or quetiapine. Since first trimester-exposure to benzodiazepines can increase the risk of cleft lip and palate, these drugs should be avoided; however, a brief trial (less than two weeks) of a benzodiazepine may be considered in women with severe anxiety.

\section{Acknowledgements}

VS has received grants from Assurex, Genome Canada, Neurocrine Biosciences, Stanley Medical Research Institute, and the Academic Medical Organization of Southwestern Ontario.

The author thanks Christine Baczynski for her help with preparing the manuscript.

\section{Disclosure}

VS has participated in advisory boards for Sunovion Pharmaceuticals and Otsuka.

\section{References}

1 Fawcett EJ, Fairbrother N, Cox ML, White IR, Fawcett JM. The prevalence of anxiety disorders during pregnancy and the postpartum period: a multivariate Bayesian meta-analysis. J Clin Psychiatry. 2019;80:18r12527.

2 Goodman JH, Watson GR, Stubbs B. Anxiety disorders in postpartum women: a systematic review and meta-analysis. J Affect Disord. 2016;203:292-331.

3 Dekel S, Ein-Dor T, Dishy GA, Mayopoulos PA. Beyond postpartum depression: posttraumatic stress-depressive response following childbirth. Arch Womens Ment Health. 2020;23:557-64.

4 Miller ES, Chu C, Gollan J, Gossett DR. Obsessive-compulsive symptoms during the postpartum period. A prospective cohort. J Reprod Med. 2013;58:115-22.

5 Zimmerman M, Martin J, McGonigal P, Harris L, Kerr S, Balling C, et al. Validity of the DSM-5 anxious distress specifier for major depressive disorder. Depress Anxiety. 2019;36:31-8. 